\section{R Maharjan1 \\ RP Shrivastav2 \\ H Bhattarai2}

Department of ENT and Head \& Neck Surgery, National Academy of Medical Science, Kathmandu, Nepal.1 Ganesh Man Singh Memorial Academy of ENT and Head \& Neck Studies, TU Teaching Hospital , Kathmandu, Nepal.2

\section{Correspondence to:}

Dr. Rupa Maharjan

Department of ENT and Head and Neck Surgery, National Academy of Medica Science, Kathmandu, Nepal. E-mail: rupa_maharjan11@yahoo.com

\title{
ANALYSIS OF TASTE DISTURBANCES IN PATIENTS BEFORE \& AFTER MASTOID SURGERY
}

Objectives:

To observe the frequency of taste disturbance in all patients undergoing mastoid surgery and to correlate between peroperative status of chorda tympani nerve (CTN) and type of peroperative CTN injury with postoperative taste disturbance.

Materials and methods:

A prospective analysis of patients who underwent mastoid surgery over 18 months period in Ganesh Man Singh Memorial Academy of ENT and Head \& Neck Studies, TU Teaching Hospital, Kathmandu, Nepal. The peroperative status of CTN and different types of peroperative CTN injuries were also noted and categorized. Subjective assessment of taste disturbance was categorized as altered taste sensation/metallic taste, tongue numbness, absence of taste sensation and dry mouth. Results:

All patients did not have any preoperative taste disturbance. On the 2 nd postoperative day (POD), taste disturbances were found in 13 patients $(11.5 \%)$, out of which 10 patients $(76.96 \%)$ had numbness and 3 patients $(23.07 \%)$ had absence of taste sensation. On the 10 th POD the finding was similar as previous. However, two months after surgery, only one patient (7.69\%) remained symptomatic. This symptomatic patient also recovered his taste sensation after 4th months of surgery. Out of these 13 patients who developed postoperative taste disturbances, in 5 patients $(38.46 \%)$ CTN was not found/not identified. Remaining 8 patients, in whom the CTN was found/identified, in 5 patients (38.46\%) it was clean cut with micro-scissors, in whom 4 patients had numbness of tongue and 1 patient had absence of taste sensation. Remaining 2 patients in whom the CTN was cut while using burr, both patients developed numbness of tongue and the single remaining patient in whom the CTN was stretched but preserved, the patient also developed numbness of tongue $(p>0.05)$.

Conclusion:

No patients in this study suffered from taste disturbance before mastoid surgery despite of the fact that, in 44 patients (39\%) the CTN was not identified at the time of surgery. Postoperatively taste disturbance was found in 13 patients $(11.50 \%)$, among them in 10 patients (76.92\%) the disturbance was in the form of numbness.

Key words: chorda tympani nerve (CTN), mastoid surgery, taste disturbance

\section{INTRODUCTION:}

Taste is one of the 5 basic special senses in the human body. Taste buds are the sense organs for taste and located in the walls of fungiform and vallate papillae of the tongue. The CTN, a branch of facial nerve carries taste sensation from the anterior $2 / 3$ of tongue. Mastoid surgery is a common surgery in otorhinolaryngology practice. The purpose of doing this surgery is to make the ear safe and dry, to prevent the complications and to preserve the hearing as far as possible.

The CTN is vulnerable to have different types of injuries during mastoid surgery, especially during elevation of the tympanomeatal flap, removal of the disease process and drilling of the posterior canal wall. The injury can range from just being stretched, clean cut or cut while using the burr. Occasionally the CTN may not be identified due to various reasons. CTN injury in mastoid surgery is common but postoperative taste disturbances have not generally been appreciated both by patients as well as by otologists., The taste disturbances can be in the form of a metallic taste, bitter, salty, sour, dryness of mouth and numbness of tongue. 6 This study will help medical personnel to counsel patients regarding taste disturbance preoperatively due to the existing disease process and postoperatively due to injury to the CTN.

\section{MATERIALS AND METHODS:}

This was a prospective, analytical and longitudinal study. The study was conducted in Ganesh Man Singh Memorial Academy of ENT and Head \& Neck Studies, Tribhuvan University Teaching Hospital (TUTH), Kathmandu, Nepal for a period of 18 months, from 1st October 2007 to 30th March 2009. Patients of age more than 12 years, either sex and undergoing all mastoid surgeries including revision surgeries were also included in this study. Excluded cases were patients with mental retardation, with altered taste due to other medical conditions like diabetic mellitus, suffering from neurological and psychiatric diseases, who had history of surgery on the tongue and salivary glands, having facial nerve palsy (preoperative and postoperative), patients on drugs that may cause taste disturbances such as: ACE inhibitors, amphetamines, benzodiazepines, ethambutol, cisplatin, carbamazepine, metformin, metronidazole, propranolol, streptomycin carboplatin, clarithromycin, beta-lactam antibiotics, sulfasalazine, tetracyclines, furosemide, nifedipine, losartan and ofloxacin. One day prior to surgery, patients were asked about any of the following type of taste disturbances: altered taste/metallic taste, numbness of tongue, dryness of mouth and absence of taste sensation. In this study subjective assessment of taste disturbance was done based on a questionnaire developed used by Nin et al 11 but with some modification. The type of CTN injury during mastoid surgery noted in this study was based on Gopalan et al 8 with certain modifications. In our study, the type of injury to the CTN during mastoid surgery were noted as: CTN found/identified or not found/not identified, stretched, deliberately cut with micro scissors or cut while using burr. Postoperatively patients were asked about their taste sensation. The first assessment of taste sensation was carried out at the time of $1 \mathrm{st}$ dressing, i.e., $2^{\text {nd }}$ post-operative day, then on 10th postoperative day and finally on 2 months after surgery. However, only those patients who had taste disturbances at the end of 2 nd months postoperatively were followed up to 4 th month.

\section{RESULTS:}

A total of 139 mastoid surgeries were done during our study period. Out of these 139 patients, 113 patients were included while 26 patients were excluded from the study. Among these 113 included patients, 65 were male and 48 were female. The mean age was 23.76 years. All 113 patients who underwent mastoid surgery did not have any preoperative taste disturbance.

Altogether 13 patients developed postoperative taste disturbance. They were classified in two groups:

Group A- Patients with postoperative taste disturbance in whom the CTN was not found.

Group B- Patients with postoperative taste disturbance in whom the CTN was found.

Out of 5 patients, belongs to Group A, 3 patients had tongue numbness

Fig: 1. Pia chart showing types of peroperative CTN injury

Types of peroperative chorda tympani nerve injury

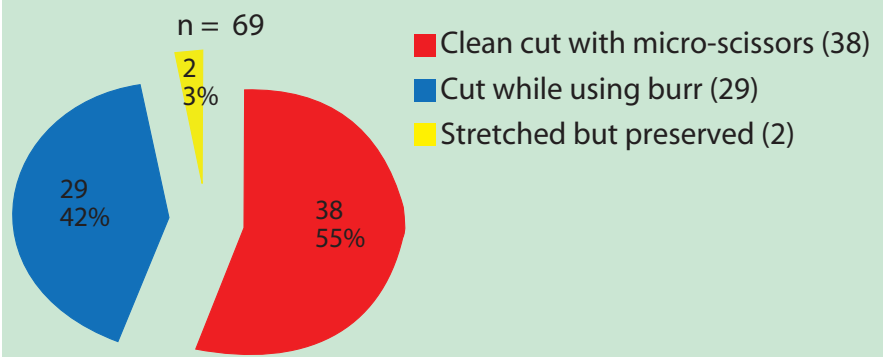


Fig: 2. Bar diagram showing postoperative taste disturbances at 1 st, 2 nd, 3 rd and at 4 th assessment

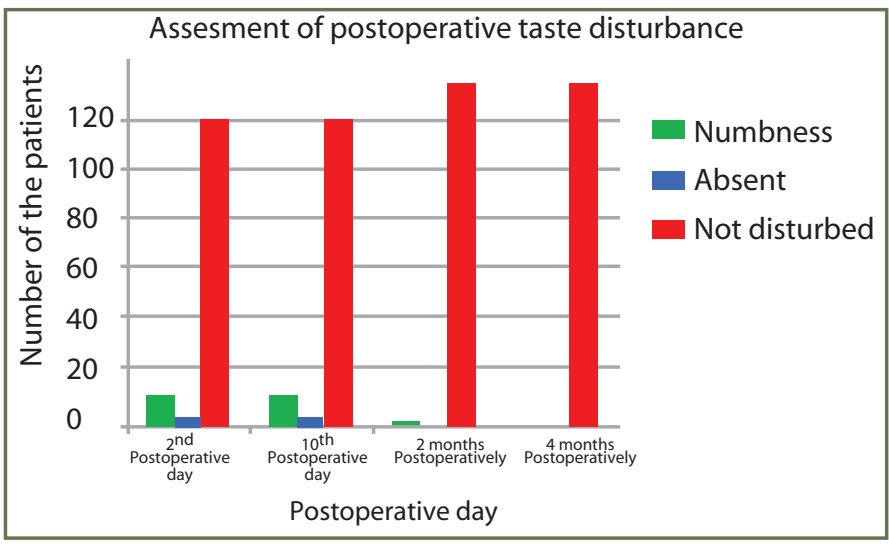

Table: 1. Status of postoperative taste sensation in relation to types of peroperat chorda tympani nerve injury and peroperative status of chorda tympani nerve

Type of peroperative CTN injury

Postoperative taste not disturbed $(n=100)$

Tongue Absence Metallic Dryness of numbness of taste taste mouth

\section{Clean cut}

with micro

- scissors

Cut while

using burr

$$
33
$$

Postoperative taste disturbance after surgery $(n=13)$

Stretched but preserved CTN not found

Total 100
27

1

1

39

4 \\ 4}

2

1

3

10

\begin{tabular}{|l|l|l|}
\hline 1 & 0 & 0 \\
\hline 0 & 0 & 0 \\
\hline 0 & 0 & 0 \\
\hline 2 & 0 & 0 \\
\hline 3 & 0 & 0 \\
\hline
\end{tabular}

where as 2 patients had absence of taste sensation. This suggests that most probably CTN was damaged preoperatively due to chronic nature of disease like COM squamous type. The other less like possibility would be the surgeon failed to identify the CTN during surgery.

Out of 8 patients, belonging to Group B, in 5 patients CTN was clean cut with micro-scissors, while in 2 patients the CTN was cut accidently by burr during drilling of bone and in 1 patient the CTN was stretched but preserved. Of these 5 patients in whom the CTN was clean cut with micro-scissors, 4 patients had numbness of tongue and 1 patient had absence of taste sensation. Of the remaining 2 patients in whom the CTN was cut while using burr, both patients developed numbness of tongue and in the last remaining 1 patient in whom the CTN was stretched but preserved, the patient developed numbness of tongue. Using Chi-Square test to correlate peroperative type of CTN injury with postoperative taste disturbance, there is no correlation between peroperative status of CTN and type of injury with postoperative taste disturbance $(p>0.05)$.

\section{DISCUSSION:}

Among the 113 studied cases, peroperative CTN was found only in 69 patients and where as it was not found in 44 patients despite of all attempts made to find it out but could not because it could be damaged preoperatively by disease, Clark et al. 6

The different types of CTN injuries, categorized in this study were: stretched, deliberately cut with micro scissors or cut while using burr or stretched but preserved. This type of categorized injury is also supported by the study done by Clark et al 6 in which out of 21 patients who underwent mastoid surgery, the CTN was sectioned in 16 patients (76.19\%) but only 5 patients (31\%) became symptomatic. Likewise, in 5 patients $(33.80 \%)$ CTN was stretched but preserved and none of them became symptomatic. In this study also the most common type of injury was clean cut with micro scissors: 38 patients $(55.07 \%)$ which was followed by cut while using burr in 29 patients (42.02\%) and stretched but preserved in 2 patients (2.89\%).

It is interesting to note here that although during surgery in 44 patients (39\%) CTN was not found, but none of these patients had taste disturbance preoperatively. That could be due to damage of the different middle ear structures like ossicles, nerve, etc by inflammatory changes 2 . Landis et al reported that changes in the taste sensation of the tongue, on the ipsilateral side of chronic inflammatory middle ear disease proceed unnoticed by the patients. That could be the reason that none of the patients included in this study complained of taste disturbance preoperatively. All patients were specifically asked for any taste disturbances as they did not voluntarily complain about it. Similar subjective assessment have been implied in other studies done by Gopalan et al7, Micheal et al8, Sone et al11 and Sakagami et al. 12

In the literature, the post- operative symptoms of taste disturbance vary from 15 to 31\%, Gopalan et al7 and Clark et al.6 In our study, frequency of CTN injury was high $61.06 \%$. However, $11.59 \%$ developed immediate postoperative taste disorder. Our study showed a comparatively lower percentage of symptomatic patients than found in rest of the literature. According to Clark et al6, because of the chronic disease process of cholesteatoma leads to gradual hypofunction and patient is not aware of further postoperative taste disturbance following CTN injury.

In our study, none of the patients were aware of symptoms until they were asked about taste disturbance specifically. Contrary to this study, in the study done by Gopalan et al7 and Mahendran et al11, patients complained of taste disturbances following middle ear surgery without being specifically asked for it.

2 In this study, $11.50 \%$ developed taste disturbance after mastoid surgery. The most common taste disturbance was numbness of tongue $76.96 \%$. Another was absence of taste $23.07 \%$. None of the patient complained of metallic taste and dry mouth. Similarly Nin et al10 also found numbness in 58\%. But Clark et al6 and Micheal et al8 found the commonest taste disturbance was metallic taste sensation.

In our study, there were 44 patients where chorda tympani nerve was not found. Among these 44 patients, 5 patients (38\%) were symptomatic even when the chorda was not found. The most like cause that can be presumed that, CTN was present and unknowingly injured during surgery. Saito et al9 suggested that the CTN could have been unknowingly touched or tugged without the surgeon being aware of its existence in the surgical field.

\section{CONCLUSION:}

None of the 113 patients with COM squamous active type were found to have taste disturbance before mastoid surgery. Postoperatively, taste disturbance was found in only $11.50 \%$. However, none of the patients voluntarily complained of postoperative taste disturbance. They complained of taste disturbance only after being asked for it by the doctor. The most frequent type of CTN injury during mastoid surgery was clean cut with micro scissors. Numbness of the tongue was the most common taste disturbance found after mastoid surgery. Taste disturbance was encountered in $38 \%$ patients in whom CTN was not found. Among the CTN identified cases, CTN was clean cut with micro scissors in 38\% patients, CTN was cut while using burr in $15 \%$ patients and CTN was stretched but preserved in $8 \%$ patients. However, these findings were statistically not significant. Hence there is no direct relation between peroperative status of CTN findings and type of CTN injury with postoperative taste disturbance.

\section{REFERENCES:}

1. Ganong WF. Smell and Taste. In: Foltin J, Matragrano J, Ransom J, Davis K, editor. Review of Medical Physiology. 21st ed. San Francisco: Mc Graw Hill, 2003:188-94.

2. Tos M. Pathology of ossicular chain in various middle ear disease. J Laryngol Otol Aug 1979;93(8):769-80. 
3. Gedikli O, Dogru H, Aydin G, Tuz M, Uygur , K Sari. Histopathological changes of chorda tympani in chronic otitis media. Laryngoscope 2001;111:724-7.

4. Landis BN, Beutner D, Fransnelli J, Huttenbrink KB, Hummel T. Laryngoscope June 2005;115(6):1124-7.

5. Kveton JF. Open Cavity Mastoid Operations. In: Card GW, editor. Glasscock Shambaugh Surgery of the Ear. 5th ed. Elsevier, 2003:499515.

6. Clark PAM, O'Malley S. Chorda tympani nerve function after middle ear surgery. Otology \& Neurotology April 2007;28(3):335-40.

7. Gopalan P, Kumar M \& Gupta M et al. A study of chorda tympani nerve injury and related symptoms following middle ear surgery. J Laryngol Otol 2005;119:189-92.

8. Micheal P, Raut V. Chorda tympani nerve injury; operative findings and operative symptoms. Otolaryngol Head and Neck surgery 2007;136(6):978-81. 